\title{
Apremilast Induces Apoptosis of Human Colorectal Cancer Cells with Mutant KRAS
}

\author{
KENSUKE NISHI ${ }^{1,2 *}$, HAO LUO $^{1 *}$, SHUHEI ISHIKURA ${ }^{1,3}$, KEIKO DOI $^{1,3}$, \\ YURI IWAIHARA ${ }^{1}$, LAUREN WILLS ${ }^{4}$, GEORGE S. BAILLIE $^{4}$, TOSHIFUMI SAKATA ${ }^{2}$, \\ SENJI SHIRASAWA ${ }^{1,3}$ and TOSHIYUKI TSUNODA ${ }^{1,3}$ \\ Departments of ${ }^{1}$ Cell Biology, and ${ }^{2}$ Otorhinolaryngology, Faculty of Medicine, and \\ ${ }^{3}$ Central Research Institute for Advanced Molecular Medicine, Fukuoka University, Fukuoka, Japan; \\ ${ }^{4}$ Institute of Cardiovascular and Medical Science, University of Glasgow, Glasgow, Scotland, U.K.
}

\begin{abstract}
Background/Aim: We previously reported the crucial roles of oncogenic Kirsten rat sarcoma viral oncogene homologue (KRAS) in inhibiting apoptosis and disrupting cell polarity via the regulation of phosphodiesterase type $4 B 2$ (PDE4B2) expression in human colorectal cancer (CRC) HCT116 cells in a three-dimensional culture (3DC). Here, we evaluated the effects of apremilast, a selective PDE4 inhibitor, on luminal apoptosis in 3DC and nude mice assay using HKe3 human CRC cells stably expressing wild-type (wt)PDE4B2 (HKe3-wtPDE4B2), mutant (mt)PDE4B2 (kinase dead) (HKe3-wtKRAS), wtKRAS (HKe3-wtKRAS) and mtKRAS (HKe3-mtKRAS). Materials and Methods: Apoptosis was detected by immunofluorescence using confocal laser scanning microscopy or western blot in HKe3-wtPDE4B2, HKe3mtPDE4B2, HKe3-wtKRAS and mtKRAS cells treated with or without apremilast in 3DC. Tumourigenicity was assessed in nude mice assay using these cells. Results: Apremilast did not inhibit the proliferation of HKe3-wtPDE4B2 cells or HKe3mtKRAS in two-dimensional cultures, whereas the number of apoptotic HKe3-wtPDE4B2 cells and HKe3-mtKRAS cells increased after apremilast treatment in $3 D C$, leading to formation of a luminal cavity. Tumour growth in nude mice was dramatically reduced by intraperitoneal injection of apremilast. Notably, a decreased level of caspase-1 expression was observed in HKe3-wtPDE4B2 and HKe3-mtKRAS cells.
\end{abstract}

This article is freely accessible online.

*These Authors contributed equally to this study.

Correspondence to: Toshiyuki Tsunoda, MD, Ph.D., Department of Cell Biology, Faculty of Medicine Fukuoka University, 7-45-1 Nanakuma, Jonan-ku, Fukuoka 814-0180, Japan. Tel: +81 928011011, Fax: +81 928643865, e-mail: tsunoda@fukuoka-u.ac.jp

Key Words: Apremilast, apoptosis, colorectal cancer, KRAS, PDE4B2.
Conclusion: Apremilast induces tumour regression in nude mice, possibly by inducing caspase-1 expression.

The impairment of cyclic AMP (cAMP) generation by overexpression of phosphodiesterase 4 (PDE4) isoforms, including PDE4A, PDE4B, PDE4C and PDE4D (1, 2), has been described in various cancer pathologies (3). Indeed, many recent studies have indicated that PDE4 inhibitors are effective against different types of cancer cells (4-9). In our recent studies, we found that $\mathrm{PDE} 4 \mathrm{~B}$ is specifically upregulated by mutated Kirsten rat sarcoma viral oncogene homologue (KRAS) in three-dimensional (3D) culture, and PDE4 inhibitors, including rolipram and resveratrol, were found to induce luminal apoptosis of human colorectal cancer cells with mutated KRAS in 3D culture $(10,11)$, suggesting that PDE4 is a novel effector protein downstream of mutated KRAS signaling within the in vivo microenvironment. Currently, there are no effective drugs that directly target effectors of KRAS signaling or mutated $K R A S$ in clinical use. Therefore, PDE4 inhibitors may provide a novel strategy for targeting different types of cancer cells with mutated $K R A S$, including colorectal and pancreatic cancers.

Apremilast, an oral small molecule inhibitor of PDE4, is approved by the United States Food and Drug Administration for treating patients with chronic inflammatory disorders, such as psoriasis and psoriatic arthritis $(12,13)$. Notably, apremilast shows favourable adverse reaction profiles compared to other PDE inhibitors, which are milder than emesis with cilomilast (14) and central nervous system effects with rolipram (15), suggesting its appropriateness for cancer treatment and chemoprevention. Our study intended to determine the effects of apremilast in cancer with mutated KRAS (in vivo and in vitro). For this reason, we performed 3D floating culture and nude mice assays using the HKe3 cells overexpressing wild type (wt) PDE4B2, mutated (mt) PDE4B2 (kinase dead) mtKRAS and wtKRAS. Our results 
suggest that apremilast may be an effective treatment for human colorectal cancer.

\section{Materials and Methods}

Antibodies and reagents. Antibody to cleaved caspase-3 (5A1), cleaved caspase-7 (D6H1), cleaved poly (ADP-ribose) polymerase (PARP) (Asp214; D64E10) and caspase-1 were obtained from Cell Signaling Technology (Beverly, MA, USA). Antibody to actin was obtained from Sigma-Aldrich (St. Louis, MO, USA). Apremilast was a kind gift from Celgene Corp, NJ, USA.

Retroviral production and generation of stable cell line. Dasher Green Fluorescent Protein (DGFP) cDNA from DNA2.0's Cas9 vectors ( $\mathrm{pD} 1401-\mathrm{AD}$ ) was subcloned into a pMSCVpuro vector (Clontech, Palo Alto, CA, USA) at a multi-cloning site to generate retrovirus vector to produce a pMSCV-DGFP vector. cDNAs for Hemagglutinin (HA)-tagged human wtPDE4B2 (wtPDE4B2-HA) and HA-tagged human mtPDE4B2 (H234S, mt PDE4B2-HA) were inserted into the pMSCV-DGFP vector at multi-cloning sites to generate the mammalian expression plasmids pMSCV-wtPDE4B2HA-DGFP and pMSCV-mtPDE4B2-HA-DGFP, which were produced by the transfection of the retrovirus vectors together with the pAmpho vector (Clontech) into GP2-293 packaging cells (Clontech) by standard calcium phosphate transfection in the presence of $25 \mu \mathrm{M}$ chloroquine (Sigma-Aldrich). After 48 hours since transfection, the viral supernatants were collected and supplemented with $8 \mu \mathrm{g} / \mathrm{ml}$ polybrene (Sigma-Aldrich). The HKe3 cells in six-well plates were infected with the viruses by being spun for $2 \mathrm{~h}$ at $32^{\circ} \mathrm{C}$ and $1,000 \times g$. At $48 \mathrm{~h}$ after infection, the cells were treated with $2 \mu \mathrm{g} / \mathrm{ml}$ puromycin (Sigma-Aldrich) for 1 week to establish HKe3-derived cells stably expressing wtPDE4B2 or mtPDE4B2. The cells were further maintained in the medium containing $2 \mu \mathrm{g} / \mathrm{ml}$ puromycin.

Cell culture. Cells were cultured in Dulbecco's modified Eagle's medium (DMEM)-high glucose supplemented with $10 \%$ fetal bobine serum and $1 \%$ penicillin/streptomycin in the presence or absence of apremilast $(100 \mu \mathrm{M})$ for 6 days in two-dimensional (2D) or $3 \mathrm{D}$ culture as previously described $(10,16-23)$.

Cell growth assay in $2 D$ culture. Herein, $4 \times 10^{4}$ cells for HKe3wtPDE4B2, HKe3-mtPDE4B2 and HKe3-wtKRAS, and $1 \times 10^{4}$ cells for HKe3-mtKRAS cells, which were established earlier (23), were seeded in a 96-well microplate (product number CLS3596; Corning Inc., Corning, NY, USA) at $37^{\circ} \mathrm{C}$ for six days, and the absorbance of formazan solutions was measured using a 3-(4,5-dimethlthiazol2-yl)-2,5-diphenyltetrazolium bromide (MTT) assay-based Cell Counting Kit-8 (Dojindo, Kumamoto, Japan) according to the supplier's instructions. The relative growth rate was calculated from a comparison of the absorbance obtained from cells treated with dimethyl sulfoxide (DMSO) alone as a vehicle control.

$3 D$ floating cell culture. Cells were seeded in a 96-well plate with an ultra-low attachment surface and a round bottom (product number 7007; Corning Inc., Corning, NY, USA). Cells were cultured for 6 days with or without apremilast in a $\mathrm{CO}_{2}$ incubator, as previously described (23). Photomicrographs of cells were taken and analysed using an IN Cell Analyzer 1000 (GE Healthcare, Little Chalfont, UK) and IN Cell Developer Toolbox (GE Healthcare).
The relative growth rate was calculated from a comparison of the area of control spheroids at day three.

Quantification of apoptotic cells in spheroids. Apoptotic cells were stained with Magic Red Caspase $3 \& 7$ Assay Kit (ImmunoChemistry Technologies, LLC, Bloomington, MN, USA) on day 6, according to the supplier's instructions, and imaged using CS-SP5 laser scanning confocal microscopy (Leica, Wetzlar, Germany). The number of apoptotic cells in spheroids was counted as previously described $(10,18)$.

Assay for tumourigenicity. Four-week-old female SHO mice ( $\mathrm{Crl}$ : HA-Prkdcscid $H r$ hr) were purchased from Charles River Laboratories (Yokohama, Japan). Cells for implantation were trypsinised and resuspended in a 1:1 mix of phosphate-buffered saline and Matrigel (BD Bioscience, Bedford, MA, USA). $100 \mu \mathrm{l}$ containing either $1.5 \times 10^{6} \mathrm{HKe} 3-\mathrm{mtKRAS}$ or $1 \times 10^{7}$ of HKe-3-wtPDE4B2, HKe-3mtPDE4B2 or HKe3-wtKRAS cells was subcutaneously injected into the flank of mice. At the beginning of apremilast treatment, the average diameter of these tumours was approximately $5 \mathrm{~mm}$. Apremilast was intraperitoneally administered from day 0 to day 7 daily. Tumour volumes were calculated using the formula: $\left(L \times W^{2}\right)$ $\times 0.5$, where $L$ was the tumour length and $W$ the tumour width. The tumour volume at day $n$ was expressed as the relative tumour volume (RTV) and calculated according to the following formula: $\mathrm{RTV}=\mathrm{TV} n / \mathrm{TV} 0$, where $\mathrm{TV} n$ was the tumour volume at day $n$ and TV 0 the tumour volume at day 0 . Animal studies were conducted in accordance with our institutional guidelines, and the experimental procedures were approved by the Fukuoka University Animal Care Committee (approval number 1611991).

Immunoblotting. Cells treated with or without apremilast were lysed in a RIPA buffer [(50 mM Tris-HCl, pH 7.5, $150 \mathrm{mM} \mathrm{NaCl,} 1 \% \mathrm{NP}-$ $40,0.5 \%$ sodium deoxycholate, $0.1 \%$ sodium dodecyl sulphate and protease inhibitor cocktail) (Roche, Basel, Switzerland)] and were subjected to immunoblotting as previously described $(10,24)$. Quantitative analysis of the immunoblotting was performed using ImageJ software (National Institute of Health, Bethesda, MD, USA).

Statistical analyses. Data are presented as means \pm standard deviations. Statistical analyses were performed using unpaired twotailed Student's $t$-test. $p$-Values of less than 0.05 were considered statistically significant.

\section{Results}

Induction of luminal apoptosis by apremilast in HKe3wtPDE4B2 and HKe3-mtKRAS cells grown in 3D culture. To address the effects of apremilast on cell proliferation in $3 \mathrm{D}$ culture, cells were treated with apremilast or DMSO alone. No significant differences were observed in the growth rates between HKe3-wtPDE4B2 cells, HKe3-mtPDE4B2 cells or HKe3-wtKRAS cells treated with apremilast and those treated with DMSO (Figure 1A). Interestingly, however, the relative growth rate of $\mathrm{HKe} 3-\mathrm{mtKRAS}$ cells treated with apremilast was lower than in those treated with DMSO alone (Figure 1A), suggesting that apremilast, via its inhibitory action on PDE4, regulates the signalling pathway altered by mutated KRAS in 3D culture. 
A

Control $\square$ Apremilast $(100 \mu \mathrm{M})$

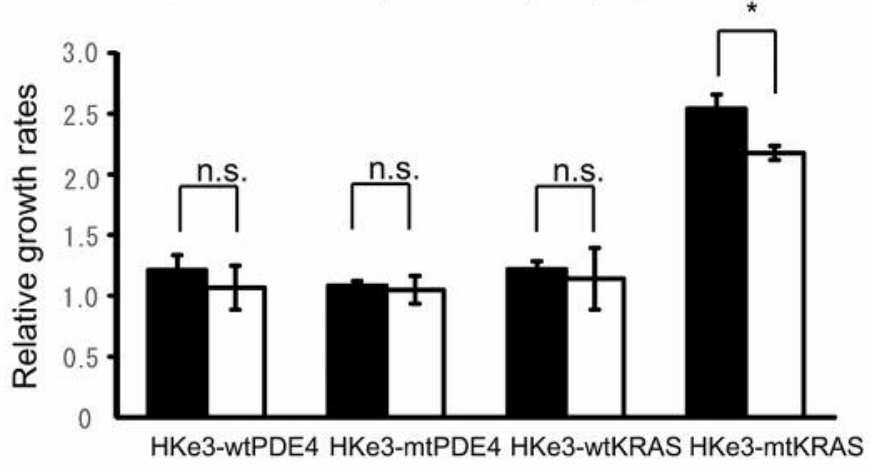

B

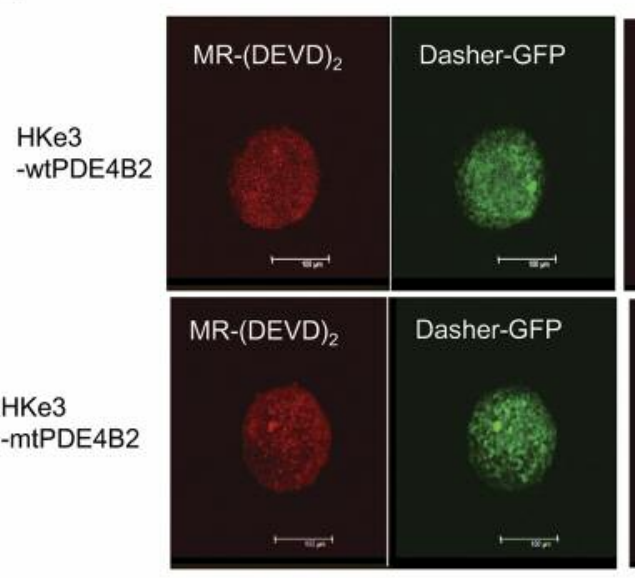

C

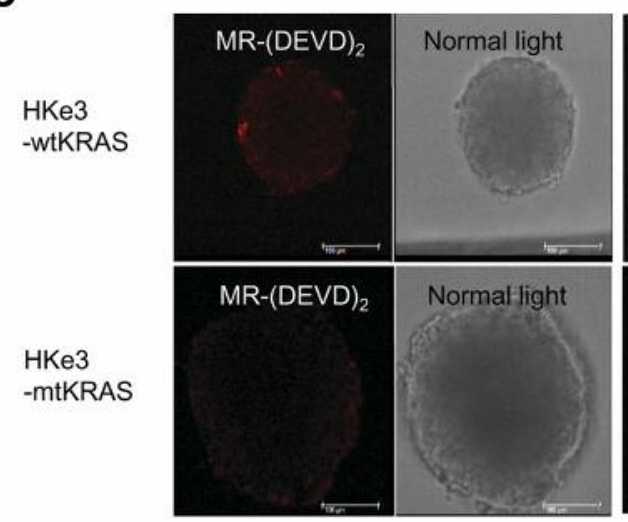

Apremilast
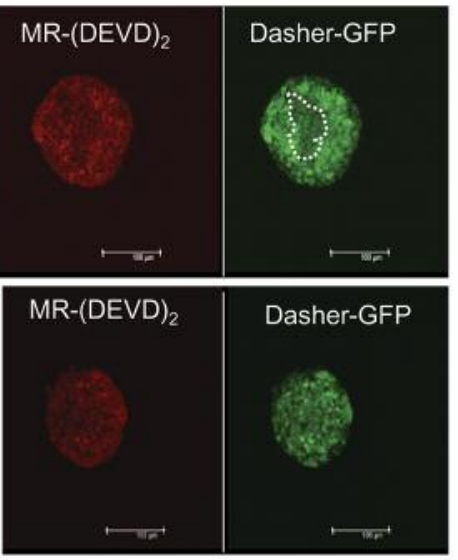

Apremilast
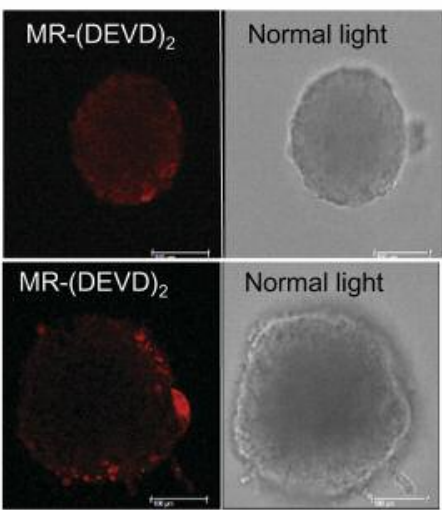

- Control $\square$ Apremilast(100 $\mu \mathrm{M})$

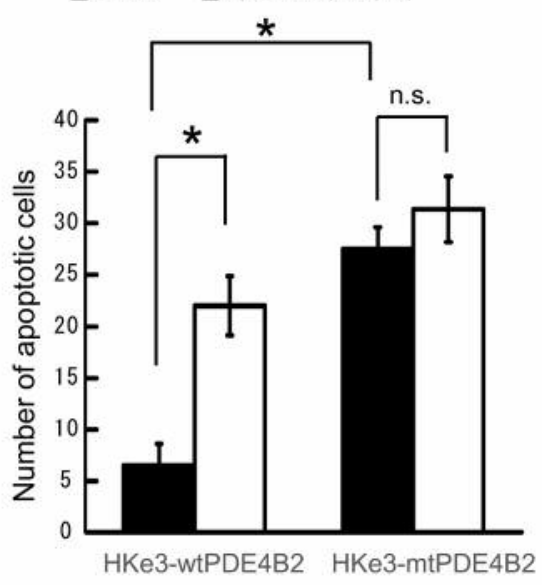

Control $\square$ Apremilast(100 $\mu \mathrm{M})$

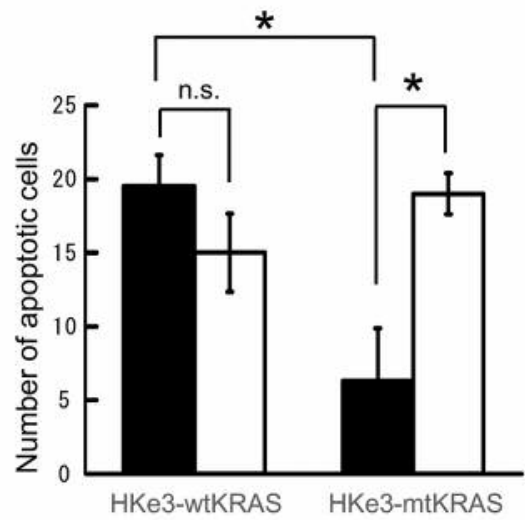

Figure 1. Luminal apoptosis was induced by apremilast in human colorectal cancer HKe3 cells stably expressing wild-type phosphodiesterase $4 B 2$ (PDE4B2) (HKe3-wtPDE4B2) and mutant Kirsten rat sarcoma viral oncogene homologue (KRAS) (HKe3-mtKRAS) cells grown in 3D culture. A: Relative growth rates of HKe3-wtPDE4B2, HKe3-mutant (mt) PDE4B2, HKe3-wtKRAS and HKe3-mtKRAS with or without apremilast treatment in $3 D$ culture at day 6. B, C: Left panels: The signals for cleaved caspase-3/7 in HKe3-wtPDE4B2 and HKe3-mtPDE4B2 cells (B) and HKe3wtKRAS and HKe3-mtKRAS $(C)$ treated with dimethyl sulfoxide alone (control) or apremilast at day 6 in 3D culture. Cleaved caspase-3/7, red. Dasher GFP, green. Dots represent luminal cavities. Scale bar=100 $\mu m$. Right panels: Number of apoptotic cells in single spheroids. *Significantly different at $p<0.05 ;$ n.s., not significant. 
To address whether the influence of apremilast extended to luminal apoptosis in $3 \mathrm{D}$ culture, we evaluated the apoptotic activity in these cells grown in 3D culture for 6 days by evaluating cleaved caspase-3/7 using confocal microscopy. In HKe3-wtPDE4B2 control cells, the number of apoptotic cells was 4.23-fold lower compared to HKe3mtPDE4B2 control cells (Figure 1B; $p<0.05$ ), suggesting that the overexpression of wtPDE4B2 was able to inhibit luminal apoptosis, whereas mtPDE4B2 (kinase dead) was not. In HKe3-wtPDE4B2 cells treated with apremilast, the number of apoptotic cells increased 3.38-fold in comparison to the DMSO control (Figure $1 \mathrm{~B} ; p<0.05$ ). In addition to increased apoptosis, a luminal cavity was observed in cells treated with apremilast, suggesting that apremilast directly inhibits the effect of wtPDE4B2. In HKe3-mtPDE4B2 cells, no significant difference was observed between DMSO control and apremilast-treated cells (Figure 1B), suggesting that apremilast acts in this system by inhibiting the PDE activity of PDE4B2. The signals for dasher GFP representing the localisation of wtPDE4B2 or mtPDE4B2 were similar, suggesting that PDE4B2 localisation was not affected by PDE4B2 mutation (Figure 1B). These results suggest that apremilast induces luminal apoptosis in colon cancer cells by inhibiting the activity of PDE4B2.

In HKe3-mtKRAS control cells, the number of apoptotic cells decreased 3.08-fold in comparison with HKe3-wtKRAS control cells (Figure 1C; $p<0.05$ ), suggesting that the overexpression of mtKRAS was able to inhibit luminal apoptosis, whereas that of wtKRAS was not. In HKe3mtKRAS cells treated with apremilast, the number of apoptotic cells increased 3-fold in comparison with DMSO alone (Figure $1 \mathrm{C} ; p<0.05$ ), suggesting that apremilast directly counteracts the oncogenic effects of mtKRAS. In HKe3-wtKRAS cells, no significant difference was observed between DMSO control and apremilast (Figure 1C), suggesting that apremilast targets signaling downstream of mtKRAS, possibly affecting cAMP dynamics orchestrated by PDE4B2. The data presented here suggests that apremilast may represent a new therapeutic avenue for colon cancer as the PDE4 inhibitor induces luminal apoptosis by inhibiting the activity of PDE4B2.

Apremilast inhibits growth of tumours expressing wtPDE4B2 and mtKRAS in nude mice. For HKe3-wtPDE4B2 cells, tumour volume experienced a 2.65-fold increase in comparison to those in HKe3-mtPDE4B2 from day 0 to day 8 (Figure 2A and $\mathrm{B}$ ), suggesting that wtPDE4B2 promotes tumour growth in vivo. Tumour volume was dramatically reduced in HKe3-wtPDE4B2 tumours in mice treated with apremilast in comparison to those administered DMSO alone (Figure 2A). In contrast, no difference was observed between HKe3-mtPDE4B2 cell tumours treated with apremilast and DMSO controls (Figure 2B), suggesting that apremilast acts via PDE4 inhibition activity in vivo. In HKe3-mtKRAS cells, tumour volume increased 9.04-fold in comparison with that in HKe3-wtKRAS from day 0 to day 8 (Figure 2C and D). Tumour volume of HKe3-mtKRAS tumours treated with apremilast was dramatically reduced than in those treated with DMSO controls (Figure 2D); conversely, no difference was observed between apremilast-treated mice with $\mathrm{HKe} 3-$ wtKRAS tumours and DMSO-treated controls (Figure 2C), suggesting that apremilast induces apoptosis by inhibiting the activity of PDE4B2 regulated by mtKRAS in vivo.

mtKRAS and PDE4B2 prevent apoptosis through downregulation of caspase-1. In order to understand the underlying signaling events regulated by PDE4B2 in a 3D environment, caspases were examined by immunoblotting in cells from 2D and 3D culture (Figure 3). In 3D culture, the amount of cleaved capase-3, caspase-7 and PARP were detected robustly in HKe3-wtKRAS and HKe3-mtPDE4B2 cells and were decreased in HKe3-mtKRAS or HKe3-wtPDE4B2 cells, suggesting that mtKRAS and PDE4B2 are involved in the inhibition of luminal apoptosis in $3 \mathrm{D}$ culture. Surprisingly, the expression of full-length caspase-1 was strongly increased by wtKRAS and mtPDE4B2 and was reduced by mtKRAS and wtPDE4B2 in 3D culture, suggesting that mtKRAS and PDE4B2 down-regulate the protein expression of caspase- 1 .

\section{Discussion}

In this study, we demonstrated for the first time that the recently approved PDE4 inhibitor apremilast significantly promoted apoptosis of HKe3-mtKRAS and HKe3wtPDE4B2 cells via a signalling route that includes PDE4B2 and caspase-1. Our previous work showed that mtKRAS prevents luminal apoptosis via the induction of PDE4B2 in HCT116 cells (18), and PDE4B2 inhibitors, including rolipram and resveratrol, induce luminal apoptosis $(10,11)$ by a previously undefined signalling route. Apremilast is known to be highly specific for PDE4 and mediates its effects in monocytes and T-cells via protein kinase A (PKA) and nuclear factor kappa B pathways (13). PDE4 is the predominant cAMP-degrading enzyme expressed in inflammatory cells in diseases, such as psoriasis (25). The most common downstream effector of cAMP is PKA (26), and PKA action can result in upregulation of caspase-1 expression (27). Hence, it is logical to suggest that regulation of cAMP dynamics by PDE4B could appropriately regulate local PKA activity to affect caspase-1 expression. Our results showed that PDE4B and mtKRAS down-regulate the expression of caspase- 1 and promote tumour growth (Figures 2 and 3). In agreement with this result, recent work has shown that caspase-1deficient mice demonstrate increased colonic epithelial cell 
A

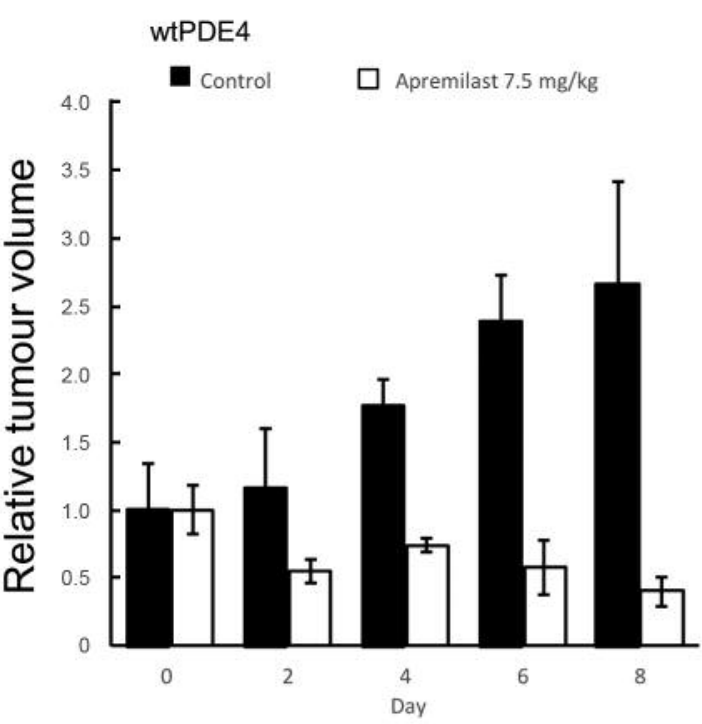

C

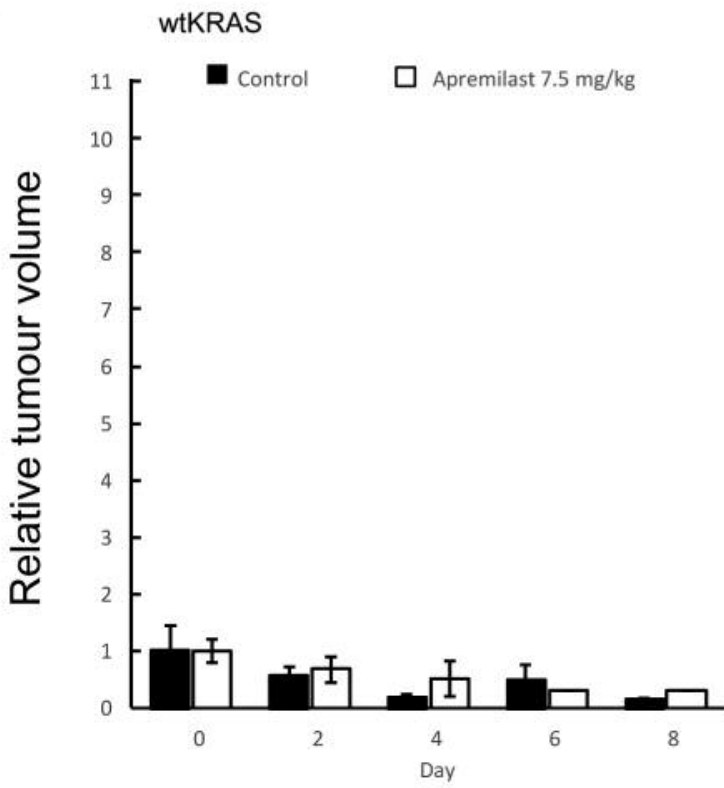

B

mtPDE4

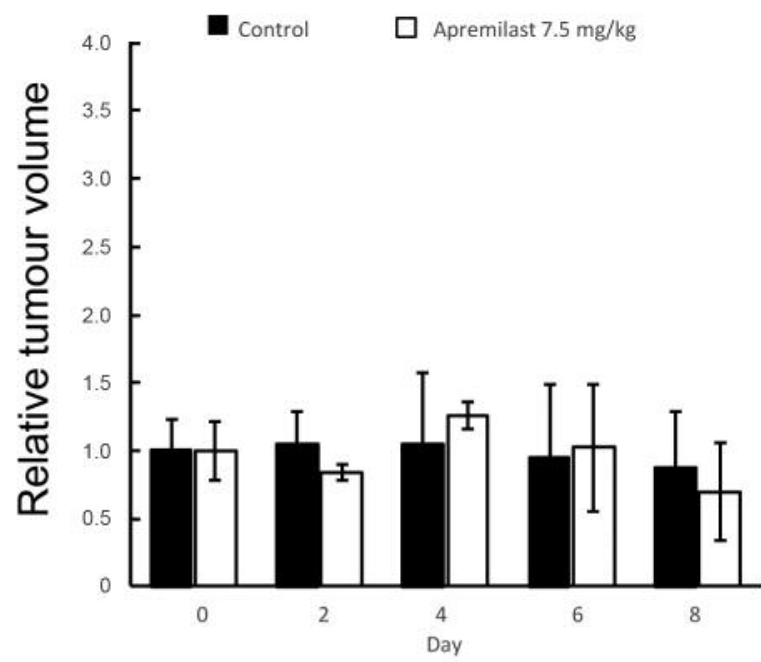

D

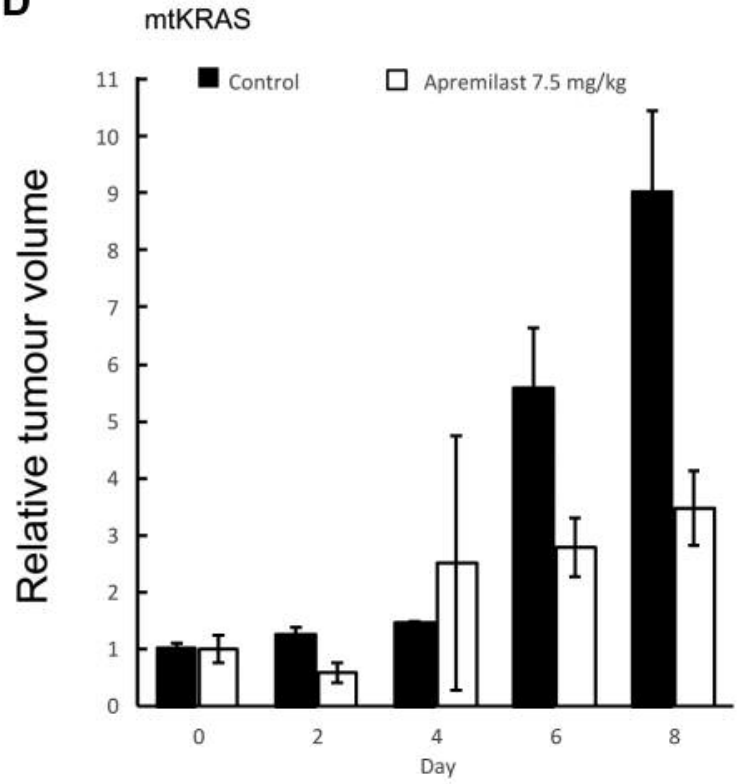

Figure 2. Assays of in vivo tumourigenicity for human colorectal cancer HKe3 cells. HKe 3 cells stably expressing wild-type phosphodiesterase $4 B 2$ (PDE4B2) (HKe3-wtPDE4B2) (A), mutant PDE4B2 (HKe3-mtPDE4B2) (B), wild-type Kirsten rat sarcoma viral oncogene homologue (KRAS) (HKe3-wtKRAS) (C) and mutant KRAS (HKe3-mtKRAS) (D) were injected subcutaneously into the flanks of nude mice. Relative tumour volumes are shown for mice treated with and without apremilast.

proliferation in early stages of injury-induced tumour formation and reduced apoptosis in advanced tumours (28). Furthermore, recent reports also suggest that caspase- 7 and not caspase- 3 is activated by caspase- 1 in vitro and in vivo $(29,30)$, and caspase- 7 is a substrate of caspase- 1 that cleaves PARP (31). In our study, the expression changes of caspase- 7 by mtKRAS or PDE4B were larger than those of caspase-3 (Figure 3). These results together suggest that mtKRAS and PDE4B are protective against apoptosis by blocking the caspase-1-caspase-7 axis. We also propose that caspase-1 signalling may offer novel downstream targets for apremilast which underpin the mechanism driving apoptosis of colon cancer cells. Further elucidation of the molecular mechanisms triggered by novel PDE4 


\section{Cleaved CASP3}

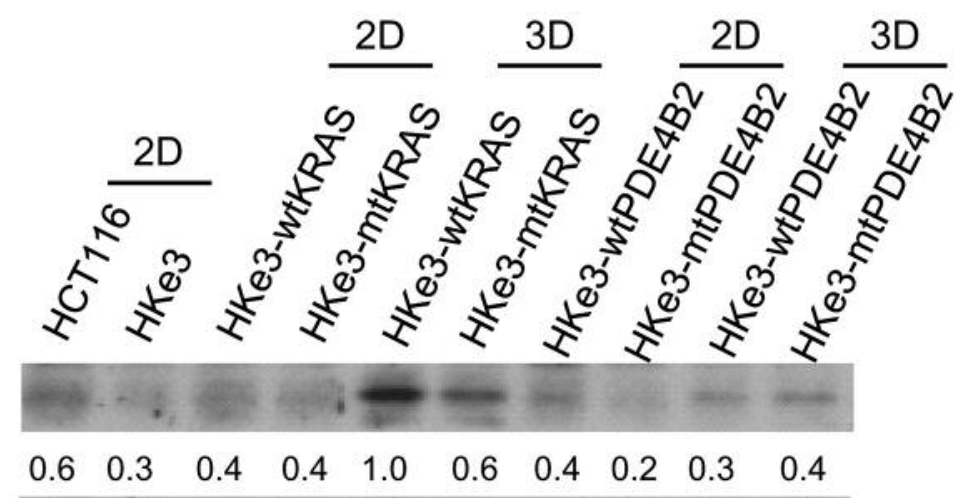

\section{Cleaved CASP7}

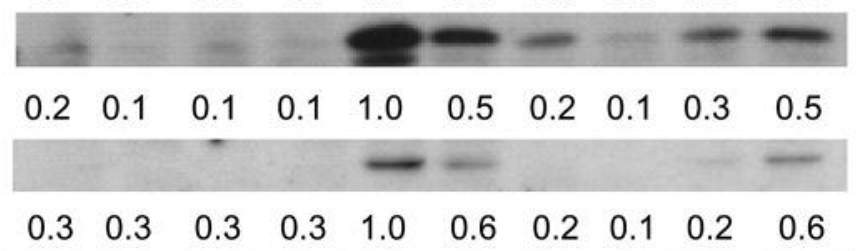

CASP1 full length (p50)

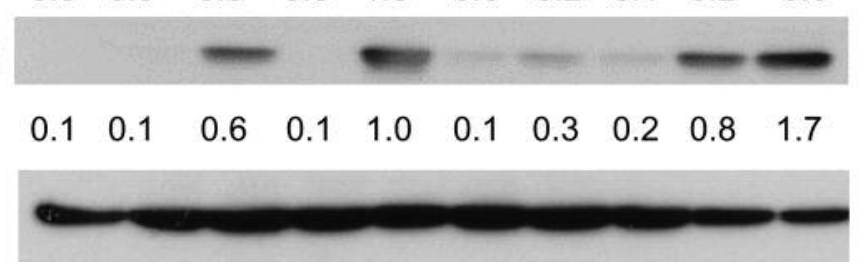

Figure 3. Mutant ( $m t$ ) Kirsten rat sarcoma viral oncogene homologue (KRAS) and wild-type (wt) phosphodiesterase 4B2 (PDE4B2) prevent apoptosis through down-regulation of caspase-1. Human colorectal cancer HKe3 cells stably expressing wtPDE4B2 (HKe3-wtPDE4B2), mtPDE4B2 (HKe3mtPDE4B2), wtKRAS (HKe3-wtKRAS) and mtKRAS (HKe3-mtKRAS) cells grown in $2 D$ or $3 D$ culture were lysed. Expression of the indicated proteins in the lysates was examined by immunoblotting using specific antibodies. Actin was used as a loading control. Levels of proteins were quantified by densitometry and normalised to actin levels. The values represent the relative ratio to levels in HKe3-wtKRAS grown in $3 D$ culture. Data are representative of three independent experiments.

inhibitors (32) such as apremilast, which drives apoptosis or pyroptosis is required to bolster the notion that PDE4 inhibition is a future therapeutic avenue for the treatment of cancer.

\section{Acknowledgements}

The Authors thank Takami Danno, Shiori Yamano and Yumiko Hirose for their technical assistance. This study was supported by the Ministry of Education, Culture, Sports, Science and Technology of Japan and the grants for UK-Japan collaboration in medicine and health (Butterfield Awards).

\section{References}

1 Spina D: PDE4 inhibitors: current status. Br J Pharmacol 155: 308-315, 2008.

2 Erdogan S and Houslay MD: Challenge of human Jurkat T-cells with the adenylate cyclase activator forskolin elicits major changes in cAMP phosphodiesterase (PDE) expression by upregulating PDE3 and inducing PDE4D1 and PDE4D2 splice variants as well as down-regulating a novel PDE4A splice variant. Biochem J 321(Pt 1): 165-175, 1997.
3 Savai R, Pullamsetti SS, Banat GA, Weissmann N, Ghofrani HA, Grimminger F and Schermuly RT: Targeting cancer with phosphodiesterase inhibitors. Expert Opin Investig Drugs 19: 117-131, 2010.

4 Marquette A, Andre J, Bagot M, Bensussan A and Dumaz N: ERK and PDE4 cooperate to induce RAF isoform switching in melanoma. Nat Struct Mol Biol 18: 584-591, 2011.

5 Kim SW, Rai D, McKeller MR and Aguiar RC: Rational combined targeting of phosphodiesterase $4 \mathrm{~B}$ and SYK in DLBCL. Blood 113: 6153-6160, 2009.

6 Smith PG, Wang F, Wilkinson KN, Savage KJ, Klein U, Neuberg DS, Bollag G, Shipp MA and Aguiar RC: The phosphodiesterase PDE4B limits cAMP-associated PI3K/AKTdependent apoptosis in diffuse large B-cell lymphoma. Blood 105: 308-316, 2005.

7 Meyers JA, Su DW and Lerner A: Chronic lymphocytic leukemia and $\mathrm{B}$ and $\mathrm{T}$ cells differ in their response to cyclic nucleotide phosphodiesterase inhibitors. J Immunol 182: 54005411, 2009.

8 Sengupta R, Sun T, Warrington NM and Rubin JB: Treating brain tumors with PDE4 inhibitors. Trends Pharmacol Sci 32: 337-344, 2011.

9 Chen TC, Wadsten P, Su S, Rawlinson N, Hofman FM, Hill CK and Schonthal AH: The type IV phosphodiesterase inhibitor 
rolipram induces expression of the cell cycle inhibitors p21(Cip1) and p27(Kip1), resulting in growth inhibition, increased differentiation, and subsequent apoptosis of malignant A-172 glioma cells. Cancer Biol Ther 1: 268-276, 2002.

10 Ota T, Doi K, Fujimoto T, Tanaka Y, Ogawa M, Matsuzaki H, Kuroki M, Miyamoto S, Shirasawa S and Tsunoda T: KRAS upregulates the expression of miR-181a, miR-200c and miR-210 in a three-dimensional-specific manner in DLD-1 colorectal cancer cells. Anticancer Res 32: 2271-2275, 2012.

11 Tsunoda T, Ishikura S, Doi K, Matsuzaki H, Iwaihara $\mathrm{Y}$ and Shirasawa S: Resveratrol induces luminal apoptosis of human colorectal cancer HCT116 cells in three-dimensional culture. Anticancer Res 34: 4551-4555, 2014.

12 Schett G, Sloan VS, Stevens RM and Schafer P: Apremilast: a novel PDE4 inhibitor in the treatment of autoimmune and inflammatory diseases. Ther Adv Musculoskelet Dis 2: 271-278, 2010 .

13 Schafer PH, Parton A, Capone L, Cedzik D, Brady H, Evans JF, Man HW, Muller GW, Stirling DI and Chopra R: Apremilast is a selective PDE4 inhibitor with regulatory effects on innate immunity. Cell Signal 26: 2016-2029, 2014.

14 Robichaud A, Stamatiou PB, Jin SL, Lachance N, MacDonald D, Laliberte F, Liu S, Huang Z, Conti M and Chan CC: Deletion of phosphodiesterase $4 \mathrm{D}$ in mice shortens alpha(2)-adrenoceptormediated anesthesia, a behavioral correlate of emesis. J Clin Invest 110: 1045-1052, 2002.

15 McCann FE, Palfreeman AC, Andrews M, Perocheau DP, Inglis JJ, Schafer P, Feldmann M, Williams RO and Brennan FM: Apremilast, a novel PDE4 inhibitor, inhibits spontaneous production of tumour necrosis factor-alpha from human rheumatoid synovial cells and ameliorates experimental arthritis. Arthritis Res Ther 12: R107, 2010.

16 Shirasawa S, Furuse M, Yokoyama N and Sasazuki T: Altered growth of human colon cancer cell lines disrupted at activated Ki-ras. Science 260: 85-88, 1993.

17 Baba I, Shirasawa S, Iwamoto R, Okumura K, Tsunoda T, Nishioka M, Fukuyama K, Yamamoto K, Mekada E and Sasazuki $\mathrm{T}$ : Involvement of deregulated epiregulin expression in tumorigenesis in vivo through activated Ki-Ras signaling pathway in human colon cancer cells. Cancer Res 60: 6886-6889, 2000.

18 Tsunoda T, Takashima Y, Fujimoto T, Koyanagi M, Yoshida Y, Doi K, Tanaka Y, Kuroki M, Sasazuki T and Shirasawa S: Threedimensionally specific inhibition of DNA repair-related genes by activated KRAS in colon crypt model. Neoplasia 12: 397-404, 2010.

19 Tsunoda T, Takashima Y, Yoshida Y, Doi K, Tanaka Y, Fujimoto T, Machida T, Ota T, Koyanagi M, Kuroki M, Sasazuki T and Shirasawa S: Oncogenic KRAS regulates miR-200c and miR$221 / 222$ in a 3D-specific manner in colorectal cancer cells. Anticancer Res 31: 2453-2459, 2011.

20 Yoshida Y, Tsunoda T, Doi K, Fujimoto T, Tanaka Y, Ota T, Ogawa M, Matsuzaki H, Kuroki M, Iwasaki A and Shirasawa S: ALPK2 is crucial for luminal apoptosis and DNA repair-related gene expression in a three-dimensional colonic-crypt model. Anticancer Res 32: 2301-2308, 2012.

21 Yoshida Y, Tsunoda T, Doi K, Tanaka Y, Fujimoto T, Machida T, Ota T, Koyanagi M, Takashima Y, Sasazuki T, Kuroki M, Iwasaki A and Shirasawa S: KRAS-mediated up-regulation of RRM2 expression is essential for the proliferation of colorectal cancer cell lines. Anticancer Res 31: 2535-2539, 2011.
22 Tsunoda T, Furusato B, Takashima Y, Ravulapalli S, Dobi A, Srivastava S, McLeod DG, Sesterhenn IA, Ornstein DK and Shirasawa S: The increased expression of periostin during early stages of prostate cancer and advanced stages of cancer stroma. Prostate 69: 1398-1403, 2009.

23 Tsunoda T, Ishikura S, Doi K, Iwaihara Y, Hidesima H, Luo H, Hirose $\mathrm{Y}$ and Shirasawa S: Establishment of a three-dimensional floating cell culture system for screening drugs targeting KRASmediated signaling molecules. Anticancer Res 35: 4453-4459, 2015.

24 Tsunoda T, Inokuchi J, Baba I, Okumura K, Naito S, Sasazuki T and Shirasawa $S$ : A novel mechanism of nuclear factor kappaB activation through the binding between inhibitor of nuclear factor-kappaBalpha and the processed $\mathrm{NH}(2)$-terminal region of Mig-6. Cancer Res 62: 5668-5671, 2002.

25 Baumer W, Hoppmann J, Rundfeldt C and Kietzmann M: Highly selective phosphodiesterase 4 inhibitors for the treatment of allergic skin diseases and psoriasis. Inflamm Allergy Drug Targets 6: 17-26, 2007.

26 Oliveira RF, Terrin A, Di Benedetto G, Cannon RC, Koh W, Kim M, Zaccolo M and Blackwell KT: The role of type 4 phosphodiesterases in generating microdomains of cAMP: large scale stochastic simulations. PLoS One 5: e11725, 2010.

27 Almeida MQ, Tsang KM, Cheadle C, Watkins T, Grivel JC, Nesterova M, Goldbach-Mansky R and Stratakis CA: Protein kinase A regulates caspase-1 via Ets-1 in bone stromal cellderived lesions: a link between cyclic AMP and proinflammatory pathways in osteoblast progenitors. Hum Mol Genet 20: 165-175, 2011.

$28 \mathrm{Hu}$ B, Elinav E, Huber S, Booth CJ, Strowig T, Jin C, Eisenbarth $\mathrm{SC}$ and Flavell RA: Inflammation-induced tumorigenesis in the colon is regulated by caspase- 1 and NLRC4. Proc Natl Acad Sci USA 107: 21635-21640, 2010.

29 Lamkanfi M, Kanneganti TD, Van Damme P, Vanden Berghe T, Vanoverberghe I, Vandekerckhove J, Vandenabeele P, Gevaert K and Nunez G: Targeted peptidecentric proteomics reveals caspase- 7 as a substrate of the caspase- 1 inflammasomes. Mol Cell Proteomics 7: 2350-2363, 2008.

30 Lamkanfi $\mathrm{M}$ and Kanneganti TD: Caspase-7: a protease involved in apoptosis and inflammation. Int J Biochem Cell Biol 42: 2124, 2010.

31 Sollberger G, Strittmatter GE, Garstkiewicz M, Sand J and Beer HD: Caspase-1: the inflammasome and beyond. Innate Immun 20: $115-125,2014$

32 Cameron RT, Coleman RG, Day JP, Yalla KC, Houslay MD, Adams DR, Shoichet BK and Baillie GS: Chemical informatics uncovers a new role for moexipril as a novel inhibitor of cAMP phosphodiesterase-4 (PDE4). Biochem Pharmacol 85: 12971305, 2013.
Received May 2, 2017

Revised May 25, 2017

Accepted May 29, 2017 